\title{
Clinical dermatology and drug discovery, relevance of secondary metabolites in Aspergillus nidulans and primary cutaneous aspergillosis
}

\author{
Calvin T Sung*, Elias Fanous, Alfred Lee, Natalia E Jacobs and John Isaac \\ School of Medicine, University of California, Riverside, Riverside, California, USA
}

Fungal secondary metabolites have been shown to be of great value in medical applications [1].

The ubiquitous Aspergillus species, in particular, has been well studied and its secondary metabolites prove to have therapeutic significance in producing antibiotics and lovastatins [2]. Aspergillus is a generic name of a group of fungi that share similar morphological characteristics that reveal spore bearing structures. Aspergillus species commonly cause localized and systemic infections, particularly in immunocompromised human hosts upon inhalation of conidia. We, unfortunately, have seen an increased number of Aspergillosis over the last decade, emphasizing the need for a better understanding of this fungus and the potentially fatal infections it may cause.

It is well documented that secondary metabolites that are produced by fungi species like Aspergillus have a wide variety of biological activities. Advances in genome sequencing have detected far more secondary metabolite gene clusters than has been previously understood. A process of attempting to activate these gene clusters are currently underway [3].

In nature, organisms produce secondary metabolites, including antibiotics that help the organism survive. Secondary metabolites are used mainly as competitive weapons against other organisms, as metal transporting agents, as symbiotic agents, as sexual hormones, and as differentiation effectors. Some secondary metabolites (including antibiotics) are important for sporulation and germination, as some secondary metabolites stimulate spore formation and inhibit or stimulate germination. In addition, sporulation and formation of secondary metabolites are regulated by similar factors, which may insure concomitant secondary metabolite production during sporulation. This serves several purposes, such as slowing down germination of spores until more favorable conditions for growth are met, protecting the spore from consumption, or cleansing the environment of competing organisms during germination.

From a clinical point of view, furthering our understanding of fungal secondary metabolites allows us to advance both drug discovery and our understanding of infectious diseases. Fungal infections commonly present with abnormal clinical findings. Much is yet to be understood in regards to how different fungal species manifest as differently as they do in immunocompetent versus immunocompromised hosts. Secondary metabolites are an area of research that has yet to be fully elucidated. Factors to consider that hinder the full understanding of secondary metabolites include the premise that they are only made in specific environmental conditions. This premise may explain the gap in understanding of how certain microbes have the ability to affect immunocompetent hosts or contaminant diseases.

Bioactive small molecules that are produced by Aspergillus species in particular have been seen to exhibit antibacterial, antifungal, and even anticancer properties. Improvements in the field such as mass spectrometry and UHPLC chromatogram utilization helps advance the drug discovery process by allowing screening measures to lead to fewer possible candidates and a more focused approach [4]. This advancement is promising in the efforts to discover more bioactive small molecules to be used for engineering novel bioactive natural products.

The medical community is often perplexed by the varied effects of fungal interactions on different individuals. This should not be surprising considering that every unique human host entails different comorbidities and underlying diseases that provide a range of environments for the fungus to invade. A better understanding of Aspergillus nidulans will provide us with a better understanding of the dermatological manifestations of Aspergillus nidulans in immunocompromised versus immunocompetent hosts, for which we currently have limited understanding.

Aspergillusisamajor fungal pathogen in severelyimmunosuppressed or neutropenic patients. Systemic Aspergillus infections occur in patients who are immunocompromised, with the most common manifestation being invasive pulmonary Aspergillosis with a mortality of $82 \%$. The skin is a rare site of infection. However, there are two cases described in the literature in which cutaneous aspergillosis was the primary manifestation of systemic aspergillosis after allogenic hematopoietic cell transplantation. For example, Kotwal, et al. reported a case of Aspergillus nidulans causing primary cutaneous aspergillosis in an immunocompetent patient [5] while Schimmelpfennig, et al. reported a case of primary cutaneous aspergillosis in a patient status post allogeneic hematopoietic cell transplantation [6]. Furthering our understanding of species such as Aspergillus and the production of their secondary metabolites is critical to the application of improving patient prognosis.

Correspondence to: Calvin T Sung, University of California, Riverside, School of Medicine, 900 University Ave, Riverside, CA 92521, USA, E-mail: calvin.sung@ medsch.ucr.edu

Received: December 10, 2017; Accepted: January 04, 2018; Published: January 10, 2018 


\section{References}

1. Keller NP, Turner G, Bennett JW (2005) Fungal secondary metabolism - from biochemistry to genomics. Nat Rev Microbiol 3: 937-947. [Crossref]

2. Vadlapudi V, Borah N, Yellusani KR, Gade S, Reddy P, et al. (2017) Aspergillus Secondary Metabolite Database, a resource to understand the Secondary metabolome of Aspergillus genus. Sci Rep 7: 7325 .

3. Demain AL, Fang A (2000) The natural functions of secondary metabolites. $A d v$ Biochem Eng Biotechnol 69: 1-39. [Crossref]
4. Bladt TT, Frisvad JC, Knudsen PB, Larsen TO (2013) Anticancer and antifungal compounds from Aspergillus, Penicillium and other filamentous fungi. Molecules 18: 11338-11376. [Crossref]

5. Kotwal A, Biswas D, Kakati B, Roy S, Chauhan BS (2015) Aspergillus nidulans causing primary cutaneous aspergillosis in an immunocompetent patient. Cutis 95: E13. [Crossref]

6. Schimmelpfennig C, Naumann R, Zuberbier T, Ordemann R, Baurmann H, et al. (2001) Skin involvement as the first manifestation of systemic aspergillosis in patients after allogeneic hematopoietic cell transplantation. Bone Marrow Transplant 27: 753-755. [Crossref]

Copyright: $\odot 2018$ Sung CT. This is an open-access article distributed under the terms of the Creative Commons Attribution License, which permits unrestricted use, distribution, and reproduction in any medium, provided the original author and source are credited. 